\title{
Invariant Properties of the Spheroidal Potential of an Oblate Planet*
}

\author{
John P. Vinti ${ }^{1}$
}

(November 17, 1965)

\begin{abstract}
The author's gravitational potential for an oblate planet, expressible in terms of oblate spheroidal coordinates, can be generalized by means of a metric-preserving transformation of the associated Cartesian system. This preserves separability of the problem of orbital motion when the potential coefficients $J_{2,1}$ and $J_{3}$ are taken into account. The inclusion of $J_{2,1}$ is not of practical importance, but has a clear physical interpretation. The inclusion of $J_{3}$, however, is of considerable practical importance, permitting a more accurate treatment than that given by perturbation theory. On the other hand, its physical significance is less clear, since including $J_{3}$ depends on translating the origin of spheroidal coordinates by a distance $\delta=\frac{1}{2} J_{2}^{-1}\left|J_{3}\right|$ equatorial radii. This distance, amounting to $7 \mathrm{~km}$ in the case of the earth, is much greater than any change in the geoid produced by $J_{3}$. It is clearly related to the long-periodic terms of perturbation theory and turns out to be equal to the displacement by $J_{3}$ of the plane of symmetry of those exactly elliptical polar orbits which are possible solutions with the spheroidal model.
\end{abstract}

Key Words: Invariant properties, spheroidal potential, oblate planet, metric-preserving transformation.

\section{Introduction}

If one neglects the axial asymmetry of an oblate planet, the gravitational potential $V$ outside it is given by

$$
V=-\frac{\mu}{r}\left[1-\sum_{n=1}^{\infty}\left(\frac{r_{e}}{r}\right)^{n} J_{n} P_{n}(\sin \theta)\right]
$$

Here $\mu$ is the product of the gravitational constant and the planet's mass, $r_{e}$ is its equatorial radius, and $r$ and $\theta$ are the planetocentric distance and latitude of a field point. The $P_{n}$ 's are the Legendre polynomials and the $J_{n}$ 's are the coefficients of potential, with $J_{2} \approx 10^{-3}$ and all higher $J^{\prime} \mathrm{s} \approx 10^{-6}$ or smaller for the earth. The first harmonic, $n=1$, can be eliminated from (1) by taking the origin at the center of mass.

If $\mathbf{r}$ is the position vector of a satellite of such a planet, its motion around the planet is described by $\ddot{\mathbf{r}}=-\nabla V$. In earlier papers ${ }^{2}$ [V1959a, b] I discovered a choice of $J_{n}$ 's that reduces the solution of this differential equation to a separable problem. This choice is

$$
J_{2 k}=(-1)^{k+1} J_{2}^{k} \quad J_{2 k+1}=(-1)^{k} J_{1} J_{2}^{k},
$$

\footnotetext{
*Research supported by the National Aeronautics and Space Administration, Washington, D.C.

1 Present address: Department of Mathematics, North Carolina State University, Raleigh, North Carolina.

${ }^{2}$ For convenience I shall use the Harvard system for references. Since there will be so many references to my own papers, however, I shall abbreviate my name to the initial $\mathrm{V}$ in the body of the paper.
} 
corresponding to the spheroidal potential

$$
V=-\frac{\mu(\rho+\eta \delta)}{\rho^{2}+c^{2} \eta^{2}}
$$

$\rho, \eta$, and $\varphi$ being spheroidal coordinates. The relations among rectangular, spherical, and spheroidal coordinates are

$$
\begin{aligned}
x+i y & =r \cos \theta \exp i \varphi=\left[\left(\rho^{2}+c^{2}\right)\left(1-\eta^{2}\right)\right]^{1 / 2} \exp i \varphi \\
z & =r \sin \theta=\rho \eta .
\end{aligned}
$$

Here

$$
c^{2}=r_{e}^{2} J_{2} \quad \delta=-r_{e} J_{1}
$$

and $\delta$ is the distance, taken positive northward, from the origin to the center of mass. Since $\delta=0$ when they coincide, this potential is symmetric with respect to the equatorial plane. Inversion of the separated equations led to an accurate reference orbit that takes into account the zeroth and second harmonic exactly and about two-thirds of the fourth harmonic [V1961a, b, 1962].

Shortly after the publication of V1959a, Brouwer and Pines [Brouwer and Clemence 1961, pp. 573, 592] found that the potential (3), with $\delta=0$, could be discovered in another way. If one imagines half the mass of the planet placed on the polar axis at $z=c^{\prime}$ and half the mass at $z=-c^{\prime}$, the corresponding two-center potential is

$$
V=-\frac{1}{2} \frac{\mu}{r_{1}}-\frac{1}{2} \frac{\mu}{r_{2}}
$$

where

$$
\left(\begin{array}{l}
r_{1}^{2} \\
r_{2}^{2}
\end{array}\right)=x^{2}+y^{2}+\left(z \mp c^{\prime}\right)^{2}
$$

This corresponds to the separable problem of two fixed centers. If one now replaces $c^{\prime}$ by $c \sqrt{-} 1$, the expression (6) remains real and leads to the separating potential (3) of the oblate planet. The significance of this procedure is traceable to the imaginary transformation that connects prolate and oblate spheroidal coordinates.

Some time later Aksenov, Grebenikov, and Demin [1963] discovered that if $\mu_{1}, \mu_{2}, r_{1}$, and $r_{2}$ are all complex, with $\mu_{1} r_{1}^{-1}$ and $\mu_{2} r_{2}^{-1}$ conjugate, the potential

$$
V=-\frac{\mu_{1}}{r_{1}}-\frac{\mu_{2}}{r_{2}}
$$

also leads to separability and enables one to fit not only $\mu$ and $J_{2}$ in (1) but also $J_{3}$, with the origin still located at the center of mass. An endeavor to understand this possibility in more physical terms led to the present paper.

\section{A Further Summary of Previous Results}

Let

$$
\xi \equiv \rho / c,
$$


where $c$ is the adjustable distance in (4.1). The potential (3) was the most general function of oblate-spheroidal coordinates that satisfied certain conditions:

a. It must be a solution of Laplace's equation.

b. It must not be infinite everywhere on the polar axis.

c. It must lead to separability of the Hamilton-Jacobi equation.

Conditions (b) and (c) imply axial symmetry, so that no explicit assumption was really necessary for that. It happened, however, that a further condition was used, but not explicitly stated:

d. The spheroidal coordinates are centered around the center of mass and around the polar axis.

\section{Transformations of the Rectangular Coordinate System}

Giving up this fourth, unstated, condition permits an extension of the results. To see how, note that the Hamiltonian can be expressed as

$$
H=\frac{1}{2} \frac{d x^{2}+d y^{2}+d z^{2}}{d t^{2}}+V(\xi, \eta)
$$

and Laplace's equation as

$$
\nabla^{2} V \equiv\left(\frac{\partial^{2}}{\partial x^{2}}+\frac{\partial^{2}}{\partial y^{2}}+\frac{\partial^{2}}{\partial z^{2}}\right) V=0
$$

Postulating that $V(\xi, \eta)$ be a potential that satisfies (10) and leads to separability, we then ask what changes one can make in the rectangular coordinate system on which $\xi, \eta, \varphi$ are based, without losing these properties. Since invariance of $d s^{2}=d x^{2}+d y^{2}+d z^{2}$ leads to invariance of $\nabla^{2}$, the answer comes easily: any metric-preserving transformation. Actually the properties are preserved even if $d s^{2}$ and $\nabla^{2}$ change by constant factors (conformal transformation). If, however, we write eqs (4) in the form

$$
\begin{aligned}
x+i y & =c\left[\left(\xi^{2}+\eta^{2}\right)\left(1-\eta^{2}\right)\right]^{1 / 2} \exp i \varphi \\
z & =c \xi \eta,
\end{aligned}
$$

it is clear that we already have a disposable scale factor, viz, $c$, so that we cannot gain any added advantage by such a generalization. Also we do not gain anything by considering reflections, since these do not introduce any extra parameters that might be used to fit more $J$ 's. Hence our practical result: to obtain the greatest generalization of the results, we need consider only translations and rotations of the rectangular coordinate system.

Now from V1959a we have the result that

$$
V=b_{0} \operatorname{Re}(\xi+i \eta)^{-1}+b_{1} \operatorname{Im}(\xi+i \eta)^{-1}
$$

is the most general potential, based on the polar axis as $z$-axis and on the center of mass as origin for the spheroidal coordinates, that satisfies conditions $a, b$, and $c$. We may now translate and rotate the associated rectangular system and (12) will still have the desired properties $a, b$, and $c$. Since a translation is described by three parameters and a rotation by three parameters, it would appear at first that we could fit six more potential coefficients. Actually the number is only five, since of the three rotation parameters, it takes only two to fix the new $z$-axis. Since the third is used to fix the rotation of the new $x$ and $y$ axes around the latter, it is immaterial with a potential like (12), which is symmetric around the new $z$-axis. 


\section{Rotation}

We assume that the polar axis is not an axis of symmetry, but that there exists such an axis $O Z$ in the planet, with angular coordinates $\psi$ and $\beta$. A plane through $O$, perpendicular to $O Z$, intersects the plane $O x y$ in the line of nodes $N$, which we take as the new $X$-axis. Then if $\mathbf{i}, \mathbf{j}$, and $\mathbf{k}$ are unit vectors along $O x, O y$, and $O z$, unit vectors $\mathbf{k}^{\prime}$ and $\mathbf{i}^{\prime}$ along $O Z$ and $O X$ are given by

$$
\begin{array}{r}
\mathbf{k}^{\prime}=\mathbf{i} \sin \psi \cos \beta+\mathbf{j} \sin \psi \sin \beta+\mathbf{k} \cos \psi \\
\mathbf{i}^{\prime} \sin \psi=\mathbf{k}^{\prime} \times \mathbf{k}=\mathbf{i} \sin \psi \sin \beta-\mathbf{j} \sin \psi \cos \beta,
\end{array}
$$

so that

$$
\mathbf{i}^{\prime}=\mathbf{i} \sin \beta-\mathbf{j} \cos \beta
$$

and

$$
\mathbf{j}^{\prime}=\mathbf{k}^{\prime} \times \mathbf{i}=\mathbf{i} \cos \psi \cos \beta+\mathbf{j} \cos \psi \sin \beta-\mathbf{k} \sin \psi .
$$

Then since

$$
\mathbf{i} x+\mathbf{j} y+\mathbf{k} z=\mathbf{i}^{\prime} X+\mathbf{j}^{\prime} Y+\mathbf{k}^{\prime} Z,
$$

we obtain the equation

$$
\left(\begin{array}{l}
x \\
y \\
z
\end{array}\right)=A\left(\begin{array}{l}
X \\
Y \\
Z
\end{array}\right)
$$

where $A$ is the square rotation matrix

$$
A=\left(\begin{array}{ccc}
\sin \beta & \cos \psi \cos \beta & \sin \psi \cos \beta \\
-\cos \beta & \cos \psi \sin \beta & \sin \psi \sin \beta \\
0 & -\sin \psi & \cos \psi
\end{array}\right)
$$

Here $O Z$ is along the axis of symmetry of $V=b_{0} \operatorname{Re}(\xi+i \eta)^{-1}+b_{1} \operatorname{Im}(\xi+i \eta)^{-1}$, having angular coordinates $\psi$ and $\beta$, the axis $O X$ is along the line of nodes, and the sense of $O Y$ is given by the righthand screw rule.

\section{Translation Plus Rotation}

If we also translate the origin from $O$ to $O^{\prime}$ by a vector

$$
\mathbf{O O}^{\prime} \equiv \mathbf{s}=\mathbf{i} s_{1}+\mathbf{j} s_{2}+\mathbf{k} s_{3}
$$

we find

$$
\left(\begin{array}{l}
x \\
y \\
z
\end{array}\right)=\left(\begin{array}{l}
s_{1} \\
s_{2} \\
s_{3}
\end{array}\right)+A\left(\begin{array}{l}
X \\
Y \\
Z
\end{array}\right)
$$


and

$$
\left(\begin{array}{l}
X \\
Y \\
Z
\end{array}\right)=A^{-1}\left(\begin{array}{l}
x-s_{1} \\
y-s_{2} \\
z-s_{3}
\end{array}\right)=\left(\begin{array}{lll}
\sin \beta & -\cos \beta & 0 \\
\cos \psi \cos \beta & \cos \psi \sin \beta & -\sin \psi \\
\sin \psi \cos \beta & \sin \psi \sin \beta & \cos \psi
\end{array}\right)\left(\begin{array}{l}
x-s_{1} \\
y-s_{2} \\
z-s_{3}
\end{array}\right)
$$

Then

$$
Z=\left(x-s_{1}\right) \sin \psi \cos \beta+\left(y-s_{2}\right) \sin \psi \sin \beta+\left(z-s_{3}\right) \cos \psi=(\mathbf{r}-\mathbf{s}) \cdot \mathbf{k}^{\prime}
$$

Also, if $\mathbf{R}$ is the position vector $\mathbf{i}^{\prime} X+\mathbf{j}^{\prime} Y+\mathbf{k}^{\prime} Z$, then $\mathbf{R}=\mathbf{r}-\mathbf{s}$ and

$$
R^{2}=r^{2}+s^{2}-2 \mathbf{s} \cdot \mathbf{r}
$$

Our separating potential is then

$$
V=b_{0} \operatorname{Re}(\xi+i \eta)^{-1}+b_{1} \operatorname{Im}(\xi+i \eta)^{-1},
$$

where

$$
\begin{aligned}
X+i Y & =c\left[\left(\xi^{2}+1\right)\left(1-\eta^{2}\right)\right]^{1 / 2} \exp i \varphi^{\prime}, \\
Z & =c \xi \eta
\end{aligned}
$$

and $\varphi^{\prime}$ is the $\varphi$-angle relative to $O^{\prime} X Y Z$.

\section{Spherical Harmonic Expansion Relative to the Original System $O x y z$}

In order to fit the coefficients of potential, we must now expand the potential $V(\xi, \eta)$ in spherical harmonics relative to the original system $O x y z$. Since we shall now obtain tesseral harmonics also, we have then to compare the expansion to be obtained with

$V=-\frac{\mu}{r}\left[1-\sum_{n=2}^{\infty}\left(\frac{r_{e}}{r}\right)^{n} J_{n} P_{n}(\cos \theta)+\sum_{n=2}^{\infty}\left(\frac{r_{e}}{r}\right)^{n} \sum_{m=1}^{n} P_{n}^{m}(\cos \theta)\left(C_{n, m} \cos m \varphi+S_{n, m} \sin m \varphi\right)\right]$.

In this section only, we use $\theta$ to mean colatitude, rather than latitude, of a field point relative to $O x y z$. This is to facilitate use of the addition theorem for spherical harmonics and because $\psi$ is the colatitude of $O^{\prime} Z$.

To obtain the expansion of $V(\xi, \eta)$ in $(20)$, we need to expand $(\xi+i \eta)^{-1}$. Now

$$
(\xi+i \eta)^{2}=\xi^{2}-\eta^{2}+2 i \xi \eta
$$

and from eqs (21)

$$
\begin{gathered}
\xi^{2}-\eta^{2}=\frac{R^{2}}{c^{2}}-1 \\
2 i \xi \eta=2 i \frac{Z}{c} .
\end{gathered}
$$

From (23), (24), (18), and (19) it then follows, if $\mathbf{s}^{2}=s^{2}$, that

$$
(\xi+i \eta)^{2}=\frac{r^{2}}{c^{2}}\left[1+\frac{s^{2}-c^{2}}{r^{2}}-2 i \frac{c}{r} \mathbf{s} \cdot \mathbf{k}^{\prime}+\frac{2}{r^{2}} \mathbf{r} \cdot\left(i c \mathbf{k}^{\prime}-\mathbf{s}\right] .\right.
$$


But

$$
\left(i c \mathbf{k}^{\prime}-\mathbf{s}\right)^{2}=s^{2}-2 i c \mathbf{s} \cdot \mathbf{k}^{\prime}-c^{2},
$$

since $\mathbf{k}^{\prime 2}=1$, so that

$$
(\xi+i \eta)^{2}=\frac{r^{2}}{c^{2}}\left[1+\frac{\left(i c \mathbf{k}^{\prime}-\mathbf{s}\right)^{2}}{r^{2}}+\frac{2}{r}\left(i c \mathbf{k}^{\prime}-\mathbf{s}\right) \cdot \mathbf{1}_{r}\right],
$$

where $\mathbf{l}_{r}$ is a unit vector along $\mathbf{r}$. Then

$$
(\xi+i \eta)^{-1}=\frac{c}{r}\left[1+\frac{2}{r}\left(i c \mathbf{k}^{\prime}-\mathbf{s}\right) \cdot \mathbf{1}_{r}+\left(\frac{i c \mathbf{k}^{\prime}-\mathbf{s}}{r}\right)^{2}\right]^{-1 / 2} .
$$

Since $c$ and $|\mathbf{s}|$ will both be small compared to $r$, we have

$$
\left|\frac{2}{r}\left(i c \mathbf{k}^{\prime}-\mathbf{s}\right) \cdot \mathbf{1}_{r}+\left(\frac{i c \mathbf{k}^{\prime}-\mathbf{s}}{r}\right)^{2}\right| \ll 1
$$

and we then have the valid binomial expansion

$$
(\xi+i \eta)^{-1}=\frac{c}{r}\left[1-\frac{1}{r}\left(i c \mathbf{k}^{\prime}-\mathbf{s}\right) \cdot \mathbf{1}_{r}-\frac{1}{2}\left(\frac{i c \mathbf{k}^{\prime}-\mathbf{s}}{r}\right)^{2}+\ldots\right] .
$$

Now the term $-c r^{-2}\left(i c \mathbf{k}^{\prime}-\mathbf{s}\right) \cdot \mathbf{1}_{r}$ will give rise to first harmonic terms unless we choose s properly. From (20) we find that the choice

$$
b_{0} \mathbf{s}-b_{1} c \mathbf{k}^{\prime}=0
$$

will eliminate terms in $r^{-2}$ from $V(\xi, \eta)$ and thus place the origin $O$ at the center of mass. Thus we must choose $\mathbf{s}$ to be either parallel or antiparallel to $\mathbf{k}^{\prime}$ (implying that the new $Z$-axis must pass through the old origin, even after the combined translation plus rotation), so that

$$
\mathbf{s}=s \mathbf{k}^{\prime} \quad(31.1), \quad b_{0} s-b_{1} c=0,
$$

where the sign of $s$ is still undetermined. Then

$$
i c \mathbf{k}^{\prime}-\mathbf{s}=(i c-s) \mathbf{k}^{\prime} \quad(32.1), \quad\left(i c \mathbf{k}^{\prime}-\mathbf{s}\right)^{2}=(i c-s)^{2}
$$

Insertion of (32) into (27) then gives

$$
(\xi+i \eta)^{-1}=\frac{c}{r}\left[1-\frac{2}{r}(s-i c) \mathbf{k}^{\prime} \cdot \mathbf{1}_{r}+\left(\frac{s-i c}{r}\right)^{2}\right]^{-1 / 2}
$$

Since

$$
\mathbf{l}_{r}=\mathbf{i} \sin \theta \cos \varphi+\mathbf{j} \sin \theta \sin \varphi+\mathbf{k} \cos \theta,
$$

it follows from (34) and (13.1) that, if $U$ is the angle between $\mathbf{k}^{\prime}$ and $\mathbf{l}_{\mathbf{r}}$,

$$
\mathbf{k}^{\prime} \cdot \mathbf{1}_{r}=\cos U=\cos \psi \cos \theta+\sin \psi \sin \theta \cos (\varphi-\beta)
$$


Then, from (33) and (35),

$$
\begin{aligned}
(\xi+i \eta)^{-1} & =\frac{c}{r}\left[1-\frac{2}{r}(s-i c) \cos U+\left(\frac{s-i c}{r}\right)^{2}\right]^{-1 / 2} \\
& =\frac{c}{r} \sum_{n=0}^{\infty}\left(\frac{s-i c}{r}\right)^{n} P_{n}(\cos U) .
\end{aligned}
$$

Insertion of the addition theorem for spherical harmonics

$$
P_{n}(\cos U)=P_{n}(\cos \psi) P_{n}(\cos \theta)+2 \sum_{m=1}^{n} \frac{(n-m) !}{(n+m) !} P_{n}^{m}(\cos \psi) P_{n}^{m}(\cos \theta) \cos (m \varphi-m \beta)
$$

into (37) then yields

$$
\begin{aligned}
(\xi+i \eta)^{-1}=\frac{c}{r} \sum_{n=0}^{\infty}\left(\frac{s-i c}{r}\right)^{n}\left[P_{n}(\cos \psi)\right. & P_{n}(\cos \theta) \\
& \left.+2 \sum_{m=1}^{n} \frac{(n-m) !}{(n+m) !} P_{n}^{m}(\cos \psi) P_{n}^{m}(\cos \theta) \cos (m \varphi-m \beta)\right] .
\end{aligned}
$$

With application of (20) and (31.2) to (39) we may readily verify that the terms involving $J_{1}, C_{1,1}$, and $S_{1,1}$ are missing from (22), so that we have indeed placed the origin at the center of mass. We began with eight disposable parameters $b_{0}, b_{1}, c, s_{1}, s_{2}, s_{3}, \psi$, and $\beta$ and after eliminating the first harmonics we are left with five, viz, $b_{0}, s, c, \psi$, and $\beta, b_{1}$ being fixed by (31.2).

On comparing (39) and (20) with (22), we next find that to fit the zeroth harmonic, we need

$$
b_{0} c=-\mu .
$$

Then

$$
b_{0}=-\frac{\mu}{c} \text { and } b_{1}=-\frac{\mu s}{c^{2}} .
$$

Let us now put

$$
s=\sigma r_{e} \cos \gamma \quad c=\sigma r_{e} \sin \gamma,
$$

so that

$$
s-i c=\sigma r_{e} \exp (-i \gamma), \quad(s-i c)^{n}=\sigma^{n} r_{e}^{n} \exp (-i n \gamma) .
$$

From (39), (20), and (22), we then find for the zonal harmonics,

$$
\mu r_{e}^{n} J_{n}=c P_{n}(\cos \psi)\left[b_{0} \operatorname{Re}(s-i c)^{n}+b_{1} \operatorname{Im}(s-i c)^{n}\right] .
$$

Application of (43) to (44) then gives

$$
J_{n}=\sigma^{\prime \prime} P_{n}(\cos \psi) \frac{\sin (n-1) \gamma}{\sin \gamma},
$$

yielding the expected results $J_{0}=-1, J_{1}=0$, and

$$
\begin{aligned}
& J_{2}=\sigma^{2} P_{2}(\cos \psi) \\
& J_{3}=2 \sigma^{3} P_{3}(\cos \psi) \cos \gamma
\end{aligned}
$$




$$
J_{4}=\sigma^{4} P_{4}(\cos \psi) \frac{\sin 3 \gamma}{\sin \gamma}
$$

For the tesseral and sectorial harmonics we find

$$
C_{n, m}+i S_{n, m}=-2 \frac{(n-m) !}{(n+m) !} \sigma^{n} P_{n}^{m}(\cos \psi) \frac{\sin (n-1) \gamma}{\sin \gamma} \exp (\operatorname{im} \beta),
$$

leading to the expected results $C_{1,1}=S_{1,1}=0$ and to

$$
\begin{aligned}
& C_{2,1}=-\frac{\sigma^{2}}{2} \sin 2 \psi \cos \beta \\
& S_{2,1}=-\frac{\sigma^{2}}{2} \sin 2 \psi \sin \beta \\
& C_{2,2}=-\frac{\sigma^{2}}{4} \sin ^{2} \psi \cos 2 \beta \\
& S_{2,2}=-\frac{\sigma^{2}}{4} \sin ^{2} \psi \sin 2 \beta .
\end{aligned}
$$

We now have four parameters remaining, $\sigma, \psi, \gamma$, and $\beta$, to be fitted to certain of the potential coefficients above. Clearly we must fit to $J_{2}$. The remaining choices are to fit to $J_{3}$ or $J_{4}$ and to $C_{2,1}$ and $S_{2,1}$ or to $C_{2,2}$ and $S_{2,2}$. Now it is clear that in any practical application $\psi$ would have to be a very small angle. Let

$$
J_{2,1}=\left(C_{2,1}^{2}+S_{2,1}^{2}\right)^{1 / 2} \quad J_{2,2}=\left(C_{2,2}^{2}+S_{2,2}^{2}\right)^{1 / 2} .
$$

From (48), $J_{2,2}$ would be of order $J_{2,1}^{2}$, making $J_{2,1}$ much larger than $J_{2,2}$. This is contrary to all experience, since for the earth $J_{2,2}$ is a measure of equatorial ellipticity and $J_{2,1}$ of the variation in latitude produced by wobbling of the polar axis. We should therefore fit not to $C_{2,2}$ and $S_{2,2}$ but to $C_{2,1}$ and $S_{2,1}$. The resulting much too small values for $C_{2,2}$ and $S_{2,2}$ simply mean that we cannot fit them by this model.

\section{The Physical Significance of the Rotation $\psi, \beta$}

As we have defined it, $\psi$ is the angle between the polar axis and the axis of symmetry of the separating potential $V=b_{0} \operatorname{Re}(\xi+i \eta)^{-1}+b_{1} \operatorname{Im}(\xi+i \eta)^{-1}$. Now nonvanishing of $\psi$ leads to nonvanishing of $J_{2,1}$, which would actually vanish if $O z$ were a principal axis of the primary body. It is thus natural to identify the axis of symmetry of $V(\xi, \eta)$ with that principal axis which is closest to the axis of rotation.

For the earth $\psi \approx 0.1^{\prime \prime}$, the mean variation of latitude associated with the wandering of the pole. For the moon it is likewise expected to be very small.

Furthermore, when we use a potential that has symmetry about some axis other than that of rotation, the potential, and thus the Hamiltonian, become explicit functions of time, if we use an inertial reference system. This circumstance would defeat the separability. There is another possibility, that of letting the system $O x y z$ be rigidly attached to the primary body, as we have actually done above. We should then have to treat the effect of rotation on the orbit of a satellite by means of apparent forces. They are not explicit functions of time, but they also destroy the separability, unless they are so small that we could neglect them. There might be some point in such a procedure for the moon, since its rotation is relatively slow, but even for the moon the apparent forces will undoubtedly be a good deal larger than those arising from $J_{2,1}$. If we could have fitted $J_{2,2}$, the story might have been different. 
All in all the rotational invariance of $V(\xi, \eta)$ does not appear to lead to any practical results. I have included a treatment of it partly to verify that we do not miss anything useful by abstaining from its use, but mostly because its physical significance is so transparently clear. When we make such a rotational transformation, the new axis that we find is an axis of symmetry of the resulting potential. This stimulates us to search for a physical meaning of the translation s, which turns out to be of real practical value, but whose physical significance is much less apparent.

\section{Results of the Translational Invariance of $V(\xi, \eta)$}

On putting $\psi=0$, we thereby eliminate the rotational transformation and obtain the results of the pure translational invariance. We then have left only two paramters, $\sigma$ and $\gamma$, to be fitted to the $J_{n}$ 's. By (45) we thus obtain

$$
J_{n}=\sigma^{n} \frac{\sin (n-1) \gamma}{\sin \gamma}
$$

leading to

$$
\begin{aligned}
& J_{2}=\sigma^{2} \\
& J_{3}=2 \sigma^{3} \cos \gamma \\
& J_{4}=\sigma^{4}\left(4 \cos ^{2} \gamma-1\right) \\
& J_{5}=4 \sigma^{5} \cos \gamma\left(2 \cos ^{2} \gamma-1\right) .
\end{aligned}
$$

We must certainly fit to $J_{2}$. Thus

$$
\sigma=J_{2}^{1 / 2}
$$

Since (51.3) and (51.5) lead to

$$
4 \cos ^{2} \gamma=\frac{J_{4}+J_{2}^{2}}{J_{2}^{2}}
$$

we could fit to $J_{4}$ only if $J_{4}+J_{2}^{2}$ were positive. Although the various investigators still find widely differing values of $J_{4}$, they now agree that $J_{4}+J_{2}^{2}$ is negative. Thus we cannot fit to $J_{4}$.

On fitting to $J_{3}$, we then find

$$
\cos \gamma=\frac{1}{2} J_{3} J_{2}^{-3 / 2}
$$

which leads by (42) and (52) to

$$
s=\frac{1}{2} r_{e} \frac{J_{3}}{J_{2}} \quad(54.1) \quad c=r_{e} J_{2}^{1 / 2}\left(1-\frac{1}{4} \frac{J_{3}^{2}}{J_{2}^{3}}\right)^{1 / 2} .
$$

With $J_{2}=(1.083) 10^{-3}$ and $J_{3} \approx-(2.4) 10^{-6}$, we find $\frac{1}{4} J_{3}^{2} J_{2}^{-3} \approx 4 \times 10^{-3}$, so that the new value for $c$ is about 2 parts in a thousand smaller than the value arrived at in V1959a. With $\psi=0$, we have $\mathrm{s}=s \mathbf{k}$, where $s \approx-7.09 \mathrm{~km}$. The values of $J_{4}$ and $J_{5}$ corresponding to the above values of $\sigma$ and $\gamma$ are 


$$
\begin{gathered}
J_{4}=-J_{2}^{2}+\frac{J_{3}^{2}}{J_{2}} \\
J_{5}=-2 J_{3}\left(J_{2}-\frac{1}{2} \frac{J_{3}^{2}}{J_{2}}\right) .
\end{gathered}
$$

The value of $J_{4}$ given by the model is thus little changed from the earlier value of $-J_{2}^{2}$ (V1959b) and the values of $J_{5}$ and all higher $J$ 's are negligibly small. The fit to the potential is thus exact through the third zonal harmonic and accounts for at least two-thirds of the fourth zonal harmonic.

\section{The New Form for the Potential}

From (20) and (41) and $\rho=c \xi$, we now have

$$
V=-\frac{\mu(\rho-\eta s)}{\rho^{2}+c^{2} \eta^{2}}
$$

If we place

$$
s=-\delta
$$

where

$$
\delta=\frac{1}{2} r_{e} \frac{\left|J_{3}\right|}{J_{2}}
$$

is the magnitude of the distance from the new origin of spheroidal coordinates to the origin, we obtain

$$
V=-\mu \frac{\rho+\eta \delta}{\rho^{2}+c^{2} \eta^{2}}
$$

This of the same form as eq (49) in V1959b and $\delta$ has the same meaning that it had there, viz, the distance northward from the origin of spheroidal coordinates to the center of mass. The difference is that in the earlier paper the origins of spheroidal coordinates and of spherical harmonics were always the same. Had I kept $\delta \neq 0$ but expanded the spheroidal potential in spherical harmonics about the center of mass, I would have been able to fit $J_{3}$ at that time. However, the more general expressions for the quartic $G(\eta)$ that appear in eq $(63)$ of the earlier paper can now be used.

The connections between rectangular, spherical, and spheroidal coordinates are now of course a little different. They become

$$
\begin{aligned}
x+i y & =r \cos \theta \exp i \varphi=\left[\left(\rho^{2}+c^{2}\right)\left(1-\eta^{2}\right)\right]^{1 / 2} \exp i \varphi \\
z & =r \sin \theta=-\delta+\rho \eta
\end{aligned}
$$

Actually the only difference is the appearance of $\delta$ in (61.2).

Previously, with $\delta=0$, a plane through the origin of spheroidal coordinates, perpendicular to the polar axis, had two important properties. It passed through the center of mass and was a plane of symmetry of the potential. Now, with $\delta \neq 0$, by (60) and (61.2), such a plane has neither of these properties. In contrast with the rotational transformation, which was to an axis of symmetry of the potential, the translation $\mathbf{s}=-\mathbf{k} \delta$, although it has the merit of enabling us to fit $J_{3}$ also, seems difficult to understand physically. We shall find later that a solution for some of the possible orbits will give us some physical grasp of the translation. 


\section{Remarks About Resonance Denominators}

Before solving for the orbits, it is desirable to discuss briefly the bearing that the present topic has on the singularities found in perturbation theories of satellite orbits. If $I$ is the inclination, the resonance denominator 1-5 $\cos ^{2} I$ has been found, by Brouwer 1959, Garfinkel 1959, Kozai 1962 , V1963, and others, to occur with numerators $J_{4}+J_{2}^{2}$ and $J_{5}$, but not with the numerator $J_{3}$. In other words they are absent if $J_{4}+J_{2}^{2}$ and the odd $J$ 's vanish. One cuuld previously understand their absence in such a case, because it corresponds, by (2), to the author's earlier spheroidal potential (3), with $\delta=0$. Such a potential, leading to separability, is not expected to lead to such resonance denominators in a perturbation theory. E.g., the solution of V1961b, V1962, gives exact secular terms and periodic terms which can be worked out to any degree of accuracy, with everything remaining finite when $1-5 \cos ^{2} I=0$. Thus Kozai 1962 was able to do a partial check of his results by seeing whether his resonance denominators all vanished for $J_{3}=J_{5}=\ldots .=0$ and $J_{4}+J_{2}^{2}=0$.

We now have a more stringent criterion for checking the accuracy of long-periodic terms in perturbation theories. The resonance denominators should all disappear when the $J$ 's are given by (50), with $\sigma=J_{2}^{1 / 2}$ and $\cos \gamma=\frac{1}{2} J_{3} J_{2}^{-3 / 2}$. I have tried to check Kozai [1962] in this way, but find the going difficult. Despite the great complexity of that paper, it may be that he has not carried terms to a sufficiently high order to make the test applicable.

\section{Remarks About the Translation Parameter $\delta$}

Since $\delta=\frac{1}{2} r_{e} J_{2}^{-1}\left|J_{3}\right| \approx 7 \mathrm{~km}$ for the earth, it is indeed a large quantity to be produced by the nonvanishing of $J_{3}$. The latter produces a maximum change in the geoid of only about $20 \mathrm{~m}$, a quantity of the order $r_{e}\left|J_{3}\right|$. To seek for some physical significance of $\delta$, I shall consider the effect that $J_{3}$ has on some particularly simple orbits, viz, orbits which would reduce to circular equatorial orbits and circular polar orbits for $V=-\mu r^{-1}$.

\section{The Special Orbits}

To find these special orbits, we can take over some results from V1959b and V1961a, without re-solving the whole problem. The generalized momenta $p_{p}$ and $p \eta$ are given by

$$
\begin{aligned}
& p_{\rho}=\frac{\rho^{2}+c^{2} \eta^{2}}{\rho^{2}+c^{2}} \dot{\rho}= \pm \frac{F(\rho)^{1 / 2}}{\rho^{2}+c^{2}} \\
& p_{\eta}=\frac{\rho^{2}+c^{2} \eta^{2}}{1-\eta^{2}} \dot{\eta}= \pm \frac{G(\eta)^{1 / 2}}{1-\eta^{2}},
\end{aligned}
$$

where $F(\rho)$ is a quartic with physical zeros $\rho_{2} \geqq \rho_{1}>0$ and $G(\eta)$ is the quartic

$$
\begin{aligned}
G(\eta) & =-\alpha_{3}^{2}+\left(1-\eta^{2}\right)\left(\alpha_{2}^{2}+2 \mu \eta \delta+2 \alpha_{1} c^{2} \eta^{2}\right) \\
& =\alpha_{2}^{2}-\alpha_{3}^{2}+2 \mu \eta \delta+\left(2 \alpha_{1} c^{2}-\alpha_{2}^{2}\right) \eta^{2}-2 \mu \eta^{3} \delta-2 \alpha_{1} c^{2} \eta^{4}
\end{aligned}
$$

In eqs (63) $\alpha_{1}$ is the energy, $\alpha_{3}$ the $z$-component of angular momentum, and $\alpha_{2}$ the separation parameter, reducing to the total angular momentum in the Keplerian case. The physical zeros of $G(\eta)$ are $\eta_{0}$ and $\eta_{1}$, with

$$
1 \geqq \eta_{0} \geqq \eta_{1} \geqq-1,
$$

since $\eta^{2} \leqq 1$. 
We introduce the definitions

$$
\begin{aligned}
a_{0} \equiv-\frac{\mu}{2 \alpha_{1}}, e_{0} \equiv\left(1+\frac{2 \alpha_{1} \alpha_{2}^{2}}{\mu^{2}}\right)^{1 / 2}, i_{0} \equiv \cos ^{-1}\left(\alpha_{3} / \alpha_{2}\right), p_{0} & \equiv a_{0}\left(1-e_{0}^{2}\right) \\
a & \equiv \frac{1}{2}\left(\rho_{1}+\rho_{2}\right), e \equiv \frac{\rho_{2}-\rho_{1}}{\rho_{2}+\rho_{1}}, p \equiv a\left(1-e^{2}\right) .
\end{aligned}
$$

Then for an orbit with $e=0, F(\rho)$ remains zero, and $\rho$ is constant, by (62.1):

$$
\rho=a=p
$$

From (4.14) and (4.15) of V196la, we find for an equatorial orbit that

$$
\begin{aligned}
a_{0} p_{0} & =a^{2}+3 c^{2}+O\left(J_{2}^{2}\right) \\
a_{0} & =a\left(1+\frac{c^{2}}{a^{2}}\right)+O\left(J_{2}^{2}\right) .
\end{aligned}
$$

For a polar orbit, it is clear from (61.1) that $\eta^{2}$ reaches the value unity, so that

$$
\eta_{0}=+1 \quad \eta_{1}=-1
$$

From the following paper we also have for a polar orbit

$$
e=e_{0} \quad a=a_{0} \quad p=p_{0} .
$$

If $e=0$, then $e_{0}=0$, so that $a_{0}=p_{0}$ and

$$
\rho=a=a_{0}=p_{0}=p
$$

\section{An "Equatorial" Orbit}

For the case $J_{3}=0$ and thus $\delta=0$, it follows from V196la that there is a solution corresponding to $\eta_{0}=\eta_{1}=0$, so that $\eta$ then remains zero and the orbit is confined to the equatorial plane $z=0$. For $J_{3} \neq 0$ and thus $\delta \neq 0$, such an orbit does not exist: a satellite moving in the plane $z=0$ would be driven out of that plane by the force

$$
F_{3}=\nabla\left[\mu \frac{r_{e}^{3} J_{3}}{r^{4}} P_{3}(\sin \theta)\right]
$$

arising from the third harmonic.

When $\delta \neq 0$, we see from (61) that the only circular orbit that always lies in a plane perpendicular to the polar axis is given by

$$
\rho=\text { constant } \quad \eta=\text { constant. }
$$

But $\rho$ can be constant only if $\rho_{1}=\rho_{2}$ and then

$$
\rho=a .
$$

The coordinate $\eta$ can be constant only if

$$
\eta_{0}=\eta_{1} \equiv \eta^{\prime} .
$$


To find the $z$-coordinate of this plane we must therefore find $\eta^{\prime}$. Now when $\eta_{0}=\eta_{1}=\eta^{\prime}, \eta^{\prime}$ is a double zero of $G(\eta)$ and is therefore a solution of

$$
\left(\frac{d G}{d \eta}\right)_{\eta^{\prime}}=0
$$

Thus, by (63.2) and (75), $\eta^{\prime}$ is a solution of

$$
2 \mu \delta+2\left(2 \alpha_{1} c^{2}-\alpha_{2}^{2}\right) \eta^{\prime}-6 \mu \eta^{\prime 2} \delta-8 \alpha_{1} c^{2} \eta^{\prime 3}=0 .
$$

If $\delta=0$, we know that $\eta^{\prime}=0$, so that we may now assume $\eta^{\prime}$ to be small. On using the notation in (65) and solving (76) by successive approximations, we then find

$$
\eta^{\prime}=\frac{\delta}{p_{0}+c^{2} / a_{0}}\left[1-\frac{3 \delta^{2}}{p_{0}^{2}}\right]+O\left(J_{2}^{4}\right) .
$$

Then, by (61.2), (73), and (77), the $z$-coordinate of the plane of this "equatorial" orbit is

$$
z^{\prime}=-\delta+\frac{a \delta}{p_{0}+c^{2} / a_{0}}+O\left(J_{2}^{4}\right)
$$

From (67.1) and (67.2), we now find

$$
p_{0}+\frac{c^{2}}{a_{0}}=a+\frac{3 c^{2}}{a^{2}}+O\left(J_{2}^{2}\right)
$$

so that

$$
z^{\prime}=-\delta+\frac{\delta}{1+3 c^{2} / a^{2}}+O\left(J_{2}^{3}\right)
$$

and

$$
\begin{aligned}
z^{\prime} & =-\frac{3 c^{2} / a^{2}}{1+3 c^{2} / a^{2}} \delta+O\left(J_{2}^{3}\right) \\
& =-\frac{3 c^{2}}{a^{2}} \delta+O\left(J_{2}^{3}\right),
\end{aligned}
$$

since $\delta \equiv \frac{1}{2} r_{e} J_{2}^{-1}\left|J_{3}\right|$ is of order $J_{2}$. By (54.2) we now have $c^{2}=r_{e}^{2} J_{2}+O\left(J_{2}^{2}\right)$, so that finally

$$
z^{\prime}=\frac{3}{2} \frac{r_{e}^{3}}{a^{2}} J_{3}+O\left(J_{2}^{3}\right)
$$

For close orbits this gives $z^{\prime} \approx-22 \mathrm{~m}$, a reasonable figure that is of the same order as the maximum change in the geoid produced by $J_{3}$.

As a check on the above expression, one may apply the perturbation theory for $J_{3}$ given in V1963, as applied to an orbit for which $e=0$ and $\sin I=0$. Since these quantities appear in denominators of the expressions for the variations of the Delaunay variables, one has to carry out limiting processes. It turns out that the long-periodic variations give $\delta z=0$, but that the short periodic ones give $\delta z=\frac{3}{2} r_{e}^{3} a^{-2} J_{3}$, equal to the above $z^{\prime}$. Thus the present result is in agreement with perturbation calculations. 
The result can also be verified directly from the equation $\ddot{z}=-\partial V / \partial z$. Here

$$
-V=\frac{\mu}{r}+\frac{1}{2} \mu r_{e}^{2} J_{2}\left(\frac{1}{r^{3}}-\frac{3 z^{2}}{r^{5}}\right)+\frac{1}{2} \mu r_{e}^{3} J_{3}\left(\frac{3 z}{r^{5}}-\frac{5 z^{3}}{r^{7}}\right)+\ldots .
$$

Then

$$
\ddot{z}=-\frac{\mu z}{r^{3}}+\frac{1}{2} \mu r_{e}^{2} J_{2}\left(-\frac{9 z}{\dot{r}^{5}}+\frac{15 z^{3}}{r^{7}}\right)+\frac{1}{2} \mu r_{e}^{3} J_{3}\left(\frac{3}{r^{5}}-\frac{30 z^{2}}{r^{7}}+\frac{35 z^{4}}{r^{9}}\right)+\ldots .
$$

For an orbit of constant $z$ and constant radius $a$, we have $\ddot{z}=0$ and

$$
r^{2}=a^{2}+z^{2}=a^{2}\left(1+\frac{z^{2}}{a^{2}}\right)
$$

On neglecting powers of $z$ higher than the first, we then find

$$
z=\frac{3}{2} \frac{r_{e}^{3} J_{3}}{a^{2}+\frac{9}{2} r_{e}^{2} J_{2}}=\frac{3}{2} \frac{r_{e}^{3}}{a^{2}} J_{3}+O\left(J_{2}^{3}\right),
$$

as we found before.

\section{A Polar Orbit}

Since we cannot interpret the translation parameter $\delta$ as the displacement produced by $J_{3}$ of the simplest equatorial orbit, let us now try the simplest polar orbit.

We first consider the case $\delta=0$. Then by eqs (61)

$$
x^{2}+y^{2}=\left(\rho^{2}+c^{2}\right)\left(1-\eta^{2}\right) \quad z=\rho \eta .
$$

If $e=0$, then $\rho=a$ and

$$
x^{2}+y^{2}=\left(a^{2}+c^{2}\right)\left(1-\frac{z^{2}}{a^{2}}\right),
$$

leading to

$$
\frac{x^{2}+y^{2}}{a^{2}+c^{2}}+\frac{z^{2}}{a^{2}}=1
$$

This is the equation of the coordinate spheroid $\rho=a$ and simply means that when $e=0$, the satellite's motion is restricted to it. If the orbit is polar, it is then the intersection of a meridian plane with such a spheroid. It is thus an ellipse with semiminor axis $a$, semimajor axis $\left(a^{2}+c^{2}\right)^{1 / 2}$, with center (not focus!) at the center of mass of the planet. It is not a Keplerian ellipse, since the law of areas does not hold; if $R$ is the distance from a focus to the satellite, it could not be produced by a potential $-\mu R^{-1}$ acting on the latter. That my original spheroidal potential [V1959a] leads to the existence of such an orbit was first noticed by Aksenov, Grebenikov, and Demin [1964].

By (88), this elliptic polar orbit lies between the limits

$$
z= \pm a .
$$


If we now consider the case $\delta \neq 0$ and still choose $e=0$, then $\rho=a$ as before and by eqs (61) the orbit is the intersection of a meridian plane with the spheroid

$$
\frac{x^{2}+y^{2}}{a^{2}+c^{2}}+\frac{(z+\delta)^{2}}{a^{2}}=1 \text {. }
$$

It is thus an ellipse unchanged in size or shape, but with $z$ now varying between the limits

$$
\begin{aligned}
& z_{2}=-\delta+a \\
& z_{1}=-\delta-a,
\end{aligned}
$$

by (61.2).

For $J_{3}=0$, the plane of symmetry of all such elliptic polar orbits was the plane $z=0$. For $J_{3} \neq 0$, their plane of symmetry is now

$$
z=-\delta=\frac{1}{2} r_{e} J_{2}^{-1} J_{3}
$$

By (65) and (70), the energy $\alpha_{1}=-\frac{1}{2} \mu / a_{0}$ and $a_{0}=a$, so that all such orbits of fixed $a$ have the same energy. From the conservation of energy one can show that any such orbit can be produced by first placing the satellite in the plane of symmetry, at a distance $r_{0}$ from the polar axis, and then projecting it parallel to the polar axis with initial velocity

$$
\dot{z}_{0}=(\mu / a)^{1 / 2},
$$

where

$$
a^{2}=r_{0}^{2}-c^{2}
$$

It appears that we have now arrived at a sort of physical interpretation of the translation parameter $\delta$. When we go from the separable model with $J_{3}=0$ to the improved one that fits $J_{3}$, the necessary translation of the origin of spheroidal coordinates is equal to the displacement of the plane of symmetry of the elliptic polar orbits.

A certain verification can be obtained from the perturbation theory of V1963, by placing $e=0$ and $\eta_{0}=1$ into the long-periodic terms there given. We find for the variation of $z$

$$
\delta z=\frac{1}{2} \frac{J_{3}}{J_{2}} r_{e} \sin ^{2} \psi-\frac{J_{3}}{J_{2}} r_{e} \sin l \sin g \cos \psi .
$$

Thus at either pole, with $\sin \psi= \pm 1$,

$$
\delta z=\frac{1}{2} r_{e} \frac{J_{3}}{J_{2}}
$$

corresponding exactly to the above displacement. Moreover short-periodic averaging gives

$$
\overline{\delta z}=\frac{J_{3}}{4 J_{2}} r_{e}+\frac{J_{3}}{2 J_{2}} r_{e} \sin ^{2} g
$$

and long-periodic averaging then gives

$$
\overline{\delta z}=\frac{1}{2} \frac{J_{3}}{J_{2}} r_{e},
$$

the same displacement as above. 
We may sum up the physical results as follows. If we consider the equation $\ddot{\mathbf{r}}=-\nabla V$, where $V$ includes only even zonal harmonics and where all $J_{k}$ 's higher than $J_{2}$ are much smaller, there must exist a class of polar orbits which are close to true ellipses, viz, those polar ellipses corresponding to $J_{4}=-J_{2}^{2}, J_{6}=J_{2}^{3}$, etc. All these true ellipses of a given energy generate a spheroid, and varying the energy furnishes the whole set of coordinate spheroids used in the spheroidal method. The equatorial plane is the plane of symmetry of these spheroids. It is also a plane through the center of mass and the plane of symmetry of the potential when only even zonal harmonics are included.

When we include $J_{3}$ also, if all higher zonal harmonics are small, there must again exist polar orbits which are very close to true ellipses, viz, those polar ellipses corresponding to the special choices made for the higher harmonics in the present paper. Again these new ellipses generate the new coordinate spheroids. The plane through $z=-\delta$, perpendicular to the polar axis, now does not pass through the center of mass and is now not a plane of symmetry of the potential, but it is again the plane of symmetry of the elliptical polar orbits.

\title{
15. References
}

\author{
Aksenov, E. P., Grebenikov, E. A., and Demin, V. G. (1963), Soviet Astronomy 7, 276-282. \\ Aksenov, E. P., Grebenikov, E. A., and Demin, V. G. (1964), Soviet Astronomy 16, 164-174. \\ Brouwer, D. and Clemence, G., 1961, Methods of Celestial Mechanics (Academic Press, New York and London). \\ Brouwer, D., (1959), Astron, J. 64, 378-397. \\ Garfinnel, B., (1959), Astron, J. 64, 353-367. \\ Kozai, Y., 1962, Astron. J. 67, 446-461. \\ Vinti, J. P. (1959a), Phys. Rev. Letters 3, 8. \\ Vinti, J. P. (1959b), J. Res. NBS 63B (Math. and Math. Phys.), No. 2, 105-116. \\ Vinti, J. P. (1961a), J. Res. NBS 65B (Math. and Math. Phys.), No. 3, 169-201. \\ Vinti, J. P. (1961b), Astron. J. 66, 514-516. \\ Vinti, J. P. (1962), J. Res. NBS 66B (Math. and Math. Phys.), No. 1, 5-13. \\ Vinti, J. P. (1963), J. Res. NBS 67B (Math. and Math. Phys.), No. 4, 191-222.
}

(Paper 70B1-165) 